\title{
Characterizing toluene adsorption onto carbon nanotubes for environmental applications
}

\author{
Ludovica Silvani ${ }^{\mathrm{a}}$, Alessandro Latini ${ }^{\mathrm{b}}$, Danny Reible ${ }^{\mathrm{c}}$, Marco Petrangeli Papinid ${ }^{\mathrm{d}, *}$ \\ aSapienza University of Rome, Dipartimento di Chimica, P.le Aldo Moro 5, 00185 Roma, Italy, Tel. 0039 49913948; \\ Fax: 0039 49913948; email: ludovica.silvani@uniroma1.it \\ 'Sapienza University of Rome, Dipartimento di Chimica, P.le Aldo Moro 5, 00185 Roma, Italy, Tel. 0039 49913161; \\ Fax: 0039 49913161; email: alessandro.latini@uniroma1.it \\ 'Texas Tech University, Department of Civil, Environmental, and Construction Engineering, Box 41023, Lubbock, \\ TX 79409-1023, US, Tel.8068348050; email: danny.reible@ttu.edu \\ 'Sapienza University of Rome, Dipartimento di Chimica, P.le Aldo Moro 5, 00185 Roma, Italy, Tel. 0039 49913948; \\ Fax: 0039 49913948; email: marco.petrangelipapini@uniroma1.it
}

Received 22 July 2016; Received in revised form 6 September 2016; Accepted 10 September 2016

\begin{abstract}
A B S T R A C T
Two different types of carbon nanotubes (CNTs), multi-walled and single-walled carbon nanotubes (MWCNTs and SWCNTs, respectively), have been characterized as new potential sorbents for contaminant removal from aqueous phase and can be used through different technological implementations. The performance of the materials has been evaluated in comparison with the most commonly used carbonaceous material, activated carbon (AC). Adsorption properties were evaluated by kinetic and equilibrium batch tests in aqueous solution at different salinity levels. Toluene was chosen as the reference compound to simulate the water phase dissolved portion of an oil spill. The experimental results have clearly demonstrated faster motion and higher adsorption capacity of MWCNTs and SWCNTs compared with AC. CNTs have shown very high removal efficiency for dissolved toluene, up to $30 \%$ and $90 \%$ for MWCNTs and SWCNTs, respectively. These results are very promising for the prospective use of CNTs as a potential alternative sorbent for hydrophobic organic compound (HOC) removal in environmental applications.
\end{abstract}

Keywords: Isotherm; Kinetic; Adsorption; Carbonaceous materials; Carbon nanotubes

\footnotetext{
* Corresponding author.
} 